\title{
A NOTE ON MULTIPLICATIVE TRIPLE FIBONACCI SEQUENCES
}

\author{
Satish Kumar, Hari Kishan and Deepak Gupta \\ Department of Mathematics, D.N. College Meerut (U.P.) India.
}

Keywords: Fibonacci Sequence, Multiplicative Fibonacci Sequence.

Abstract: In this paper, multiplicative triple Fibonacci sequences have been discussed. Here the results obtained by Singh et.al [3] have been extended. Some new theorems have been established.

\section{Introduction:}

Fibonacci sequences and their generalizations have several interesting properties. They have applications in several fields of science. The applications have been given in Koshy's book [9].

Atanassov [5], first introduced the concept of coupled Fibonacci sequences in 1985.

The triple Fibonacci sequence is the generalization of coupled Fibonacci sequence. Lee \& Lee [4] introduced additive triple Fibonacci sequence .They discussed several specific schemes and derived recurrent relations. Atanassov introduced new ideas for additive triple Fibonacci sequence. Singh \& Sikhwal [1], [2] discussed multiplicative coupled Fibonacci sequences and additive triple Fibonacci sequences. Singh et. al [3] presented some result of multiplicative triple Fibonacci sequences under the specific schemes. They established some theorems related to multiplicative triple Fibonacci sequences. In this note some new theorems have been established.

\section{Multiplicative Triple Fibonacci Sequences:}

Let $\left\{\alpha_{i}\right\}_{i=0}^{\infty},\left\{\beta_{i}\right\}_{i=0}^{\infty}$ and $\left\{\gamma_{i}\right\}_{i=0}^{\infty}$ be three infinite sequences and six arbitrary real numbers a, b, c, $\mathrm{d}$, e and $\mathrm{f}$ be given.

If $\alpha_{0}=\mathrm{a}, \beta_{0}=\mathrm{b}, \gamma_{0}=\mathrm{c}, \alpha_{1=\mathrm{d}}, \beta_{1}=\mathrm{e}, \gamma_{1}=\mathrm{f}$, then following nine different schemes of multiplicative triple Fibonacci sequences are as follows (Singh,et.al [3]) :

\section{First Scheme}

$\alpha_{n+2}=\beta_{n+1} \gamma_{n}$

$\beta_{n+2}=\gamma_{n+1} \alpha_{n}$

$\gamma_{n+2}=\alpha_{n+1} \beta_{n}$

Fourth Scheme

$\alpha_{n+2}=\beta_{n+1} \alpha_{n}$

$\beta_{n+2}=\gamma_{n+1} \beta_{n}$

$\gamma_{n+2}=\alpha_{n+1} \gamma_{n}$

Seventh Scheme

$\alpha_{n+2}=\alpha_{n+1} \alpha_{n}$

$\beta_{n+2}=\beta_{n+1} \beta_{n}$

$\gamma_{n+2}=\gamma_{n+1} \gamma_{n}$

\section{Second Scheme}

$\alpha_{n+2}=\gamma_{n+1} \beta_{n}$

$\beta_{n+2}=\alpha_{n+1} \gamma_{n}$

$\gamma_{n+2}=\beta_{n+1} \alpha_{n}$

Fifth Scheme

$\alpha_{n+2}=\alpha_{n+1} \gamma_{n}$

$\beta_{n+2}=\beta_{n+1} \alpha_{n}$

$\gamma_{n+2}=\gamma_{n+1} \beta_{n}$

Eighth Scheme

$\alpha_{n+2}=\beta_{n+1} \beta_{n}$

$\beta_{n+2}=\gamma_{n+1} \gamma_{n}$

$\gamma_{n+2}=\alpha_{n+1} \alpha_{n}$
Third Scheme

$\alpha_{n+2}=\alpha_{n+1} \beta_{n}$

$\beta_{n+2}=\beta_{n+1} \gamma_{n}$

$\gamma_{n+2}=\gamma_{n+1} \alpha_{n}$

Sixth Scheme

$\alpha_{n+2}=\gamma_{n+1} \alpha_{n}$

$\beta_{n+2}=\alpha_{n+1} \beta_{n}$

$\gamma_{n+2}=\beta_{n+1} \gamma_{n}$

Ninth Scheme

$\alpha_{n+2}=\gamma_{n+1} \gamma_{n}$

$\beta_{n+2}=\alpha_{n+1} \alpha_{n}$

$\gamma_{n+2}=\beta_{n+1} \beta_{n}$ 
First few terms of first scheme are as follows :

\begin{tabular}{|l|l|l|l|}
\hline $\mathrm{N}$ & $\alpha_{n}$ & \multicolumn{1}{|c|}{$\beta_{n}$} & \\
\hline 0 & $\mathrm{a}$ & $\mathrm{b}$ & $\mathrm{c}$ \\
\hline 1 & $\mathrm{~d}$ & $\mathrm{e}$ & $\mathrm{f}$ \\
\hline 2 & $\mathrm{ce}$ & $\mathrm{af}$ & $\mathrm{bd}$ \\
\hline 3 & $\mathrm{a} f^{2}$ & $\mathrm{~b} d^{2}$ & $\mathrm{c} e^{2}$ \\
\hline 4 & $b^{2} d^{3}$ & $c^{2} e^{3}$ & $a^{2} f^{3}$ \\
\hline 5 & $c^{3} e^{5}$ & $a^{3} f^{5}$ & $b^{3} d^{5}$ \\
\hline
\end{tabular}

Now we obtain some results of multiplicative triple Fibonacci sequences of second order for first and second schemes.

Theorem 1. For every integer $\mathrm{n} \geq 0$,
(a) $\prod_{k=0}^{n} \beta_{2 k+5} \gamma_{2 k+4}=\prod_{k=0}^{n} \alpha_{2 k+6}$
(b) $\prod_{k=0}^{n} \gamma_{2 k+5} \alpha_{2 k+4}=\prod_{k=0}^{n} \beta_{2 k+6}$
(c) $\prod_{k=0}^{n} \alpha_{2 k+5} \beta_{2 k+4}=\prod_{k=0}^{n} \gamma_{2 k+6}$

Proof. We prove the above result by induction method.

(a) f For $\mathrm{n}=0$, then L.H.S. $=\beta_{5} \gamma_{4}=\alpha_{6}=$ R.H.S. (By first scheme)

Thus result is true for $n=0$.

Let us assume that the result is true for some integer $n=m$. Then for $n=m+1$,

$$
\begin{array}{rlr}
\text { L.H.S. } & =\prod_{k=0}^{m+1} \beta_{2 k+5} \gamma_{2 k+4} & \\
& =\beta_{2(m+1)+5} \gamma_{2(m+1)+4} \prod_{k=0}^{m} \beta_{2 k+5} \gamma_{2 k+4} & \\
& =\alpha_{2(m+1)+6} \prod_{k=0}^{m} \alpha_{2 k+6} & \text { (By induction hypothesis) } \\
& =\prod_{k=0}^{m+1} \alpha_{2 k+6} & =\text { R.H.S. }
\end{array}
$$

Thus result is true for $n=m+1$. Hence by induction method the result is true for any positive integer n.

Similar proofs can be given for remaining parts (b) and (c).

Theorem 2. For every integer $\mathrm{n} \geq 0$,
(a) $\prod_{k=0}^{n} \beta_{3 k+5} \gamma_{3 k+4}=\prod_{k=0}^{n} \alpha_{3 k+6}$
(c) $\prod_{k=0}^{n} \alpha_{3 k+5} \beta_{3 k+4}=\prod_{k=0}^{n} \gamma_{3 k+6}$
(b) $\prod_{k=0}^{n} \gamma_{3 k+5} \alpha_{3 k+4}=\prod_{k=0}^{n} \beta_{3 k+6}$

Proof. It is easy to prove.

Theorem 3. For every integer $\mathrm{n} \geq 0$,
(a) $\prod_{k=0}^{n} \beta_{l k+5} \gamma_{l k+4}=\prod_{k=0}^{n} \alpha_{l k+6}$
(c) $\prod_{k=0}^{n} \alpha_{l k+5} \beta_{l k+4}=\prod_{k=0}^{n} \gamma_{l k+6}$
(b) $\prod_{k=0}^{n} \gamma_{l k+5} \alpha_{l k+4}=\prod_{k=0}^{n} \beta_{l k+6}$

Proof. We prove the above result by induction method.
(a) For $n=0$, then
L.H.S. $=\beta_{5} \gamma_{4}=\alpha_{6}=$ R.H.S. (By first scheme)

Thus result is true for $n=0$.

Let us assume that the result is true for some integer $n=m$. Then for $n=m+1$,

$$
\begin{aligned}
& \text { L.H.S. }=\prod_{k=0}^{m+1} \beta_{l k+5} \gamma_{l k+4} \quad \beta_{l(m+1)+5} \Psi_{l(m+1)+4} \\
& =\quad \alpha_{l(m+1)+6} \prod_{k=0}^{m} \alpha_{l k+6} \\
& =\quad \prod_{k=0}^{m+1} \alpha_{l k+6} \quad=\text { R.H.S. } \\
& \prod_{k=0}^{m} \beta_{l k+5} \gamma_{l k+4}
\end{aligned}
$$

Thus result is true for $n=m+1$. Hence by induction method the result is true for any positive integer $n$.

Similar proofs can be given for remaining parts (b) and (c). 
Theorem 4. For every integer $n \geq 0$,
(a) $\prod_{k=0}^{n} \beta_{2 k+3} \gamma_{2 k+2}=\prod_{k=0}^{n} \alpha_{2 k+4}$
(c) $\prod_{k=0}^{n} \alpha_{2 k+3} \beta_{2 k+2}=\prod_{k=0}^{n} \gamma_{2 k+4}$
(b) $\prod_{k=0}^{n} \gamma_{2 k+3} \alpha_{2 k+2}=\prod_{k=0}^{n} \beta_{2 k+4}$

Proof. We prove the above result by induction method.
(a) For $\mathrm{n}=0$, then
L.H.S. $=\beta_{3} \gamma_{2}=\alpha_{4}=$ R.H.S.
(By first scheme)

Thus result is true for $n=0$.

Let us assume that the result is true for some integer $\mathrm{n}=m$. Then for $\mathrm{n}=\mathrm{m}+1$,
L.H.S. $=\prod_{k=0}^{m+1} \beta_{2 k+3} \gamma_{2 k+2} \quad \beta_{2(m+1)+3} \gamma_{2(m+1)+2}$ $\prod_{k=0}^{m} \beta_{2 k+3} \gamma_{2 k+2}$
$=\alpha_{2(m+1)+4} \prod_{k=0}^{m} \alpha_{2 k+4}$
$=\prod_{k=0}^{m+1} \alpha_{2 k+4}$
(By induction hypothesis) $=$ L.H.S.

Thus result is true for $\mathrm{n}=\mathrm{m}+1$. Hence by induction method the result is true for any positive integer n.

Similar proofs can be given for remaining parts (b) and (c).

Theorem 5. For every integer $\mathrm{n} \geq 0$,

(a) $\prod_{k=0}^{n} \beta_{2 k+4} \gamma_{2 k+3}=\prod_{k=0}^{n} \alpha_{2 k+5}$

(c) $\prod_{k=0}^{n} \alpha_{2 k+4} \beta_{2 k+3}=\prod_{k=0}^{n} \gamma_{2 k+5}$

$\prod_{k=0}^{n} \gamma_{2 k+4} \alpha_{2 k+3}=\prod_{k=0}^{n} \beta_{2 k+5}$

Proof. It is easy to prove.

Theorem 6. For every integer $\mathrm{n} \geq 0, \quad l \geq 1$
(a) $\quad \prod_{k=0}^{n} \beta_{2 k+(l+1)} \gamma_{2 k+l}=\prod_{k=0}^{n} \alpha_{2 k+(l+2)}$
(b) $\prod_{k=0}^{n} \gamma_{2 k+(l+1)} \alpha_{2 k+l}=\prod_{k=0}^{n} \beta_{2 k+(l+2)}$
(c) $\quad \prod_{k=0}^{n} \alpha_{2 k+(l+1)} \beta_{2 k+l}=\prod_{k=0}^{n} \gamma_{2 k+(l+2)}$

Proof. We prove the above result by induction method.
(a) For $n=0$, then
L.H.S. $=\beta_{(l+1)} \gamma_{l}=\alpha_{(l+2)}=$ R.H.S.
(By first Scheme)

Thus result is true for $n=0$.

Let us assume that the result is true for some integer $\mathrm{n}=\mathrm{m}$. Then for $\mathrm{n}=\mathrm{m}+1$,

$$
\begin{aligned}
\text { L.H.S. } & =\prod_{k=0}^{m+1} \beta_{2 k+(l+1)} \gamma_{2 k+l} \\
& =\beta_{2(m+1)+(l+1)} \gamma_{2(m+1)+l} \prod_{k=0}^{m} \beta_{2 k+(l+1)} \gamma_{2 k+l} \\
& =\alpha_{2(m+1)+(l+2)} \prod_{k=0}^{m} \alpha_{2 k+(l+2)} \quad \text { (By induction hypothesis) } \\
& =\prod_{k=0}^{m+1} \alpha_{2 k+(l+2)}=\text { L.H.S. }
\end{aligned}
$$

Thus result is true for $\mathrm{n}=\mathrm{m}+1$. Hence by induction method the result is true for any positive integer n. Similar proofs can be given for remaining parts (b) and (c).

Theorem 7. For every integer $\mathrm{n}, \mathrm{p} \geq 0,, \quad l \geq 1$
(a) $\quad \prod_{k=0}^{n} \beta_{l k+(p+1)} \gamma_{l k+p}=\prod_{k=0}^{n} \alpha_{l k+(p+2)}$
(b) $\prod_{k=0}^{n} \gamma_{l k+(p+1)} \alpha_{l k+p}=\prod_{k=0}^{n} \beta_{l k+(p+2)}$
(c) $\quad \prod_{k=0}^{n} \alpha_{l k+(p+1)} \beta_{l k+p}=\prod_{k=0}^{n} \gamma_{l k+(p+2)}$

Proof. We prove the above result by induction method.
(a) For $n=0$, then
L.H.S. $=\beta_{(p+1)} \gamma_{p}=\alpha_{(p+2)}=$ R.H.S.
(By first Scheme) 
Thus result is true for $n=0$.

Let us assume that the result is true for some integer $n=m$. Then for $n=m+1$,

L.H.S. $=\prod_{k=0}^{m+1} \beta_{l k+(p+1)} \gamma_{l k+p}$

$$
\begin{aligned}
& =\beta_{l(m+1)+(p+1)} \gamma_{l(m+1)+p} \prod_{k=0}^{m} \beta_{l k+(p+1)} \gamma_{l k+p} \text { (By induction hypothesis) } \\
& =\alpha_{l(m+1)+(p+2)} \prod_{k=0}^{m} \alpha_{l k+(p+2)} \\
& =\prod_{k=0}^{m+1} \alpha_{l k+(p+2) \quad \text { R.H.S. }} \quad \text {. }
\end{aligned}
$$

Thus result is true for $\mathrm{n}=\mathrm{m}+1$. Hence by induction method the result is true for any positive integer n.

Similar proofs can be given for remaining parts (b) and (c).

Theorem 8. [3].For every integer $\mathrm{n} \geq 0$,

$\alpha_{n} \beta_{n} \gamma_{n}=\left(\alpha_{0} \cdot \beta_{0} \cdot \gamma_{0}\right)^{F_{n-1}}\left(\alpha_{1} \beta_{1} \cdot \gamma_{1}\right)^{F_{n}}$

Theorem 9.[3]. For every integer $\mathrm{n} \geq 0$,

$\prod_{k=0}^{n} \alpha_{k} \beta_{k} \gamma_{k}=\left(\alpha_{0} \beta_{0} \gamma_{0}\right)^{F_{n+1}}\left(\alpha_{1} \beta_{1} \gamma_{1}\right)^{F_{n+2}-1}$

Theorem 10. For every integer $\mathrm{n} \geq 0$,

Proof. We know that

$$
\begin{gathered}
\prod_{k=0}^{n} \alpha_{k} \beta_{k} \gamma_{k}=\frac{A^{F^{n+1} B^{F_{n+2}-1}\left(1+A^{F_{n}} B^{F_{n+1}}\right)}}{1+A^{F_{n-1} B^{F}}}, \\
\text { where, } A=\alpha_{0} \beta_{0} \gamma_{0}, B=\alpha_{1} \beta_{1} \gamma_{1}
\end{gathered}
$$

$$
\begin{aligned}
\prod_{k=0}^{n} \alpha_{k} \beta_{k} \gamma_{k}= & \left(\alpha_{0} \beta_{0} \gamma_{0}\right)^{F_{n+1}}\left(\alpha_{1} \beta_{1} \gamma_{1}\right)^{F_{n+2}-1} \\
& =A^{F_{n+1}} B^{F_{n+2}-1}
\end{aligned}
$$

(By theorem 9)

Replacing $\mathrm{n}$ by $\mathrm{n}+1$, we obtain

$$
\prod_{k=0}^{n+1} \alpha_{k} \beta_{k} \gamma_{k}=A^{F_{n+2}} B^{F_{n+s}-1} \text {. }
$$

Adding (1) and (2), we get

$$
\begin{aligned}
& \prod_{k=0}^{n} \alpha_{k} \beta_{k} \gamma_{k}+\prod_{k=0}^{n+1} \alpha_{k} \beta_{k} \gamma_{k}=A^{F_{n+1}} B^{F_{n+2}-1}+A^{F_{n+2}} B^{F_{n+8}-1} \\
& \left(1+\alpha_{n+1} \beta_{n+1} \gamma_{n+1}\right) \prod_{k=0}^{n} \alpha_{k} \beta_{k} \gamma_{k}=A^{F_{n+1}} B^{F_{n+2}-1}\left(1+A^{F_{n}} B^{F_{n+1}}\right) \\
& \prod_{k=0}^{n} \alpha_{k} \beta_{k} \gamma_{k}=\frac{A^{F_{n+1} B} \bar{F}_{n+2}^{-1}\left(1+A^{F_{n}} F_{n+1}\right)}{\left(1+\alpha_{n+1} \beta_{n+1} \gamma_{n+1}\right)} \\
& =\frac{A^{F_{n+1} B^{F_{n+2}-1}\left(1+A^{F_{n}} F^{F_{n+1}}\right)}}{\left[1+\left(\alpha_{0} \cdot \beta_{0} \cdot \gamma_{0}\right)^{F_{n-1}}\left(\alpha_{1} \beta_{1} \cdot \gamma_{1}\right)^{F_{n}}\right]} \\
& =\frac{A^{F_{n+1} B^{F_{n+2}-1}\left(1+A^{F_{n}} \hat{B}_{n+1}\right)}}{\left(1+A^{F_{n-1} F^{F}}\right)}
\end{aligned}
$$

Theorem 11. For every integer $\mathrm{n} \geq 0$,

(a) $\alpha_{n+4} \beta_{n}=\gamma_{n+2}^{3}$
(b) $\beta_{n+4} \gamma_{n} \quad \alpha_{n+2}^{3}=$

(c) $\gamma_{n+4} \alpha_{n}=\beta_{n+2}^{3}$

Proof.

$$
\begin{aligned}
\text { L.H.S. } & =\alpha_{n+4} \beta_{n}=\left(\beta_{n+3} \gamma_{n+2}\right) \beta_{n} \\
= & \left(\gamma_{n+2} \alpha_{n+1}\right)\left(\alpha_{n+1} \beta_{n}\right) \beta_{n} \\
= & \gamma_{n+2} \alpha_{n+1}^{2} \beta_{n}^{2}=\left(\alpha_{n+1} \beta_{n}\right) \alpha_{n+1}^{2} \beta_{n}^{2} \\
= & \alpha_{n+1}^{3} \beta_{n=}^{3}=\gamma_{n+2}^{3}=\text { R.H.S. }
\end{aligned}
$$

(By first Scheme)

(By first Scheme)

(By first Scheme)

(By first Scheme)

Similar proofs can be given for remaining parts (b) and (c).

Theorem 12. For every integer $\mathrm{n} \geq 0$,

(a) $\alpha_{n} \alpha_{n+1} \alpha_{n+2}^{2}=\left(\alpha_{0} \beta_{0} \gamma_{0}\right)^{F_{n+1}}\left(\alpha_{1} \beta_{1} \gamma_{1}\right)^{F_{n+2}}$

(b) $\beta_{n} \beta_{n+1} \beta_{n+2}^{2}=\left(\alpha_{0} \beta_{0} \gamma_{0}\right)^{F_{n+1}}\left(\alpha_{1} \beta_{1} \gamma_{1}\right)^{F_{n+2}}$

(c) $\gamma_{n} \gamma_{n+1} \gamma_{n+2}^{2}=\left(\alpha_{0} \beta_{0} \gamma_{0}\right)^{F_{n+1}}\left(\alpha_{1} \beta_{1} \gamma_{1}\right)^{F_{n+2}}$

Proof. We prove the above result by induction method. 
(a) For $n=0$, then

$$
\begin{aligned}
\text { L.H.S. }=\alpha_{0} \alpha_{1} \alpha_{2}^{2} & =\alpha_{0} \alpha_{1} \alpha_{2} \alpha_{2} \\
& =\alpha_{0} \alpha_{1}\left(\beta_{1} \gamma_{0}\right) \alpha_{2} \quad \text { (By first scheme) } \\
& =\alpha_{0} \alpha_{1}\left(\beta_{1} \gamma_{0}\right)\left(\gamma_{1} \beta_{0}\right) \quad \text { (By second scheme) } \\
& =\left(\alpha_{0} \beta_{0} \gamma_{0}\right)\left(\alpha_{1} \beta_{1} \gamma_{1}\right) \\
& =\left(\alpha_{0} \beta_{0} \gamma_{0}\right)^{F_{1}}\left(\alpha_{1} \beta_{1} \gamma_{1}\right)^{F_{2}}=\text { R.H.S. } \quad \text { (By }
\end{aligned}
$$

(By theorem 8)

Thus result is true for $\mathrm{n}=0$.

Let us assume that the result is true for some integer $\mathrm{n}=\mathrm{m}$. Then for $\mathrm{n}=\mathrm{m}+1$,

$$
\begin{array}{rll}
\text { L.H.S. }=\alpha_{m+1} \alpha_{m+2} \alpha_{m+3}^{2} & =\alpha_{m+1} \alpha_{m+2} \alpha_{m+3} \alpha_{m+3} & \\
& =\alpha_{m+1} \alpha_{m+2}\left(\beta_{m+2} \gamma_{m+1}\right) \alpha_{m+3} & \text { (By first scheme) } \\
& =\alpha_{m+1} \alpha_{m+2} \beta_{m+2} \gamma_{m+1}\left(\gamma_{m+2} \beta_{m+1}\right) \quad \text { (By second scheme) } \\
& =\alpha_{m+1} \alpha_{m+2}\left(\gamma_{m+1} \alpha_{m}\right) \gamma_{m+1}\left(\alpha_{m+1} \beta_{m}\right) \beta_{m+1} \quad \text { (By first scheme) } \\
& =\alpha_{m} \alpha_{m+1} \alpha_{m+2}\left(\gamma_{m+1} \beta_{m}\right) \alpha_{m+1} \beta_{m+1} \gamma_{m+1} \\
& =\alpha_{m} \alpha_{m+1} \alpha_{m+2}\left(\alpha_{m+2}\right)\left(\alpha_{m+1} \beta_{m+1} \gamma_{m+1}\right) \\
& =\alpha_{m} \alpha_{m+1} \alpha_{m+2}^{2}\left(\alpha_{m+1} \beta_{m+1} \gamma_{m+1}\right) \\
& =\left(\alpha_{0} \beta_{0} \gamma_{0}\right)^{F_{m+1}}\left(\alpha_{1} \beta_{1} \gamma_{1}\right)^{F_{m+2}}\left(\alpha_{0} \beta_{0} \gamma_{0}\right)^{F_{m}}\left(\alpha_{1} \beta_{1} \gamma_{1}\right)^{F_{m+1}} \\
& \\
& =\left(\alpha_{0} \beta_{0} \gamma_{0}\right)^{F_{m}+F_{m+1}}\left(\alpha_{1} \beta_{1} \gamma_{1}\right)^{F_{m+2}+F_{m+1}} \\
& =\left(\alpha_{0} \beta_{0} \gamma_{0}\right)^{F_{m+2}}\left(\alpha_{1} \beta_{1} \gamma_{1}\right)^{F_{m+3}}=\text { R.H.S. }
\end{array}
$$

Thus result is true for $n=m+1$. Hence by induction method the result is true for any positive integer n.

Similar proofs can be given for remaining parts (b) and (c).

Theorem 13. For every integer $\mathrm{n} \geq 0$,
(a) $\frac{\alpha_{n+8}}{\alpha_{n}}=\gamma_{n+1}^{2}$
(b) $\quad \frac{\beta_{n+s}}{\beta_{n}}=\alpha_{n+1}^{2}$
(c) $\frac{\gamma_{n+s}}{\gamma_{n}}=\beta_{n+1}^{2}$
Proof. (a)

$$
\text { L.H.S. }=\frac{\alpha_{n+6}}{\alpha_{n+8}}=\frac{\beta_{n+5} \gamma_{n+4}}{\alpha_{n+8}}
$$$$
=\frac{\gamma_{n+4} \alpha_{n+8} \gamma_{n+4}}{\alpha_{n+8}}
$$

(By first scheme)

(By first scheme) $=\gamma_{n+4}^{2}=$ R.H.S.

Similar proofs can be given for remaining parts (b) and (c).

Theorem 14. For every integer $\mathrm{n} \geq 0, l \geq 1$
(a) $\frac{\alpha_{l n+8}}{\alpha_{l n}}=\gamma_{l n+1}^{2}$
(b) $\frac{\beta_{l n+8}}{\beta_{l n}}=\alpha_{l n+1}^{2}$
(c)
$\frac{\gamma_{l n+8}}{\gamma_{l n}}=\beta_{l n+1}^{2}$

Proof. It is easy to prove.

Theorem 15. For every integer $\mathrm{n}, \mathrm{p} \geq 0, l \geq 1$
(a) $\frac{\alpha_{l n+(p+s)}}{\alpha_{l n+p}}=\gamma_{l n+(p+1)}^{2}$
$\frac{\beta_{l n+(p+s)}}{\beta_{l n+p}}=\alpha_{l n+(p+1)}^{2}$
(c) $\frac{\gamma_{l n+(p+s)}}{\gamma_{l n+p}}=\beta_{l n+(p+1)}^{2}$

Proof. It is easy to prove.

\section{Conclusion:}

In this paper results obtained by Singh et.al [3] have been extended and some new results have been established. 


\section{References}

[1] Singh, B. and Sikhwal, O.(2010): Multiplicative coupled Fibonacci sequence and some fundamental properties, International Journal of Contemporary Mathematical Science, Vol. 5, No.5,223-230.

[2] Singh, B. and Sikhwal, O. (2010): Fibonacci-triple sequence and some fundamental properties, Tamkang Journal of Mathematics, Vol. 41, No. 4, 325-333.

[3] Singh, B. and Sikhwal, O. (2012): Multiplicative triple Fibonacci sequences, Applied Mathematical Sciences, Vol. 6, No. 52,2567-2572 .

[4] Lee, J. Z. and Lee, J.S.(1987): Some properties of the generalization of the Fibonacci sequence, The Fibonacci Quarterly, Vol. 25, No.2, 111-117.

[5] Atanassov, K.T., Atanassov, L.C. and Sasselov, D.D. (1985): A new perspective to the generalization of the Fibonacci sequence, The Fibonacci Quarterly, Vol. 23, No.3, 121-28.

[6] Atanassov, K.T. (1995): Remark on a new direction for a generalization of the Fibonacci sequence, The Fibonacci Quarterly, Vol. 33, No.3, 249-250.

[7] Atanassov, K.T., Atanassov,V., Shannon, A.G. and Turner, J.C. (2002): New visual perspective on Fibonacci numbers, World Scientific.

[8] Vorobyov, N.N. (1963): The Fibonacci numbers, D. C. Health and company, Boston.

[9] Koshy, T. (2001): Fibonacci and Lucas numbers with application, Wiley-Interscience Publication, New York . 